\title{
The story of single molecules, from hole- burning and early FM spectroscopy in solids, to super-resolution nanoscopy in cells and beyond
}

\author{
William E. Moerner* \\ Departments of Chemistry, and by courtesy, of Applied Physics, Stanford University, Stanford, CA \\ USA 94305
}

In the late 1970s and 1980s, many researchers around the world were exploring a novel optical storage concept, frequency domain optical storage, which was based on spectral hole-burning in the zero-phonon lines of impurities in solids at low temperatures. In 1989, experiments aimed at establishing the ultimate limits this optical storage idea led to the first optical detection and spectroscopy of a single molecule in the condensed phase using laser FM spectroscopy. At the unexplored ultimate limit of individual molecules, many surprises occurred where single molecules showed both spontaneous changes (blinking due to spectral diffusion) and light-driven control of emission, properties that were subsequently also observed in 1997 at room temperature with single green fluorescent protein variants. In 2006, PALM and related approaches showed that Abbe's optical diffraction limit of $\sim 200 \mathrm{~nm}$ can be circumvented to achieve super-resolution fluorescence microscopy, or nanoscopy, with relatively nonperturbative visible light. Essential to this advance is the combination of single-molecule fluorescence imaging with active control of the emitting concentration and sequential localization of single fluorophores decorating a structure. Super-resolution microscopy has opened up a new frontier in which biological structures and behavior can be observed in live cells with resolutions down to 20-40 nm and below. Examples range from protein superstructures in bacteria to bands in axons to details of the shapes of amyloid fibrils and much more. Current methods development research addresses ways to extract more information from each single molecule such as $3 \mathrm{D}$ position and orientation, and to assure not only precision, but also accuracy. Still, it is worth noting that in spite of all the interest in super-resolution microscopy of extended structures, even in the "conventional" single-molecule tracking regime where the motions of individual biomolecules are recorded in solution or in materials, much can be learned about nanoscale processes when ensemble averaging is removed.

\footnotetext{
* Corresponding author: wmoerner@ stanford.edu
} 